\title{
Simulating hard photon production with WHIZARD
}

\author{
J. Kalinowski ${ }^{1}$, W. Kotlarski ${ }^{2}$, P. Sopicki ${ }^{1}$, A. F. Żarnecki ${ }^{1, a}$ \\ ${ }^{1}$ Faculty of Physics, University of Warsaw, Warsaw, Poland \\ ${ }^{2}$ Institut für Kern- und Teilchenphysik, TU Dresden, Dresden, Germany
}

Received: 2 May 2020 / Accepted: 13 June 2020 / Published online: 17 July 2020

(C) The Author(s) 2020

\begin{abstract}
One of the important goals of the proposed future $\mathrm{e}^{+} \mathrm{e}^{-}$collider experiments is the search for dark matter particles using different experimental approaches. The most general search approach is based on the mono-photon signature, which is expected when production of the invisible final state is accompanied by a hard photon from initial state radiation. Analysis of the energy spectrum and angular distributions of those photons can shed light on the nature of dark matter and its interactions. Therefore, it is crucial to be able to simulate the signal and background samples in a uniform framework, to avoid possible systematic biases. The WHIZARD program is a flexible tool, which is widely used by $\mathrm{e}^{+} \mathrm{e}^{-}$collaborations for simulation of many different "new physics" scenarios. We propose the procedure of merging the matrix element calculations with the lepton ISR structure function implemented in WHIZARD. It allows us to reliably simulate the mono-photon events, including the two main Standard Model background processes: radiative neutrino pair production and radiative Bhabha scattering. We demonstrate that cross sections and kinematic distributions of mono-photon in neutrino pair-production events agree with corresponding predictions of the $\mathcal{K} \mathcal{K} \mathrm{MC}$, a Monte Carlo generator providing perturbative predictions for SM and QED processes, which has been widely used in the analysis of LEP data.
\end{abstract}

\section{Introduction}

The search for dark matter (DM) particles is one of the main research goals of many running and planned experiments. The evidence for the existence of DM has so far been based only on observations of its gravitational interactions. Still, it is expected that it can also be produced at colliders, interact with ordinary matter in direct search detectors or produce

This work was carried out in the framework of the CLICdp Collaboration.

a e-mail: Filip.Zarnecki@fuw.edu.pl (corresponding author) signal detectable in indirect search experiments when annihilating in the dense regions of the universe. Many different theoretical models have been proposed to describe the nature of DM and they result also in many different discovery scenarios. Collider experiments, assuming DM particles can be produced at high energy collisions, have to rely on indirect signatures, as direct observation of DM particle in the detector is not possible. Experimental searches are based on the processes in which the DM particle production is associated (due to production mechanism or in the decay chain of more massive objects) with the production of particular final state objects like jets, massive gauge bosons or high energy photons.

The mono-photon signature is one of the considered scenarios to look for DM particle production in future $\mathrm{e}^{+} \mathrm{e}^{-}$ colliders. We usually assume that DM particles, denoted $\chi$ in the following, can be pair produced in the $\mathrm{e}^{+} \mathrm{e}^{-}$collisions via exchange of a new mediator particle, which couples to both Standard Model (SM) particles and DM states:

$\mathrm{e}^{+} \mathrm{e}^{-} \rightarrow \chi \chi$

However, if this is the case, the produced final state is invisible in the detector. The simplest way to detect this process is via the observation of additional hard photon radiation from initial state leptons, as shown in Fig. 1. Production of the initial state radiation (ISR) photon should be independent on the details of the DM production model. By studying the distribution of photons emitted in the process

$\mathrm{e}^{+} \mathrm{e}^{-} \rightarrow \chi \chi \gamma$

we should be able to constrain the DM particle production cross section. Bounds on DM production processes with the mono-photon signature have been derived from LEP results [1-4]. Production of DM particles in this channel has also been recently considered for ILC running at $500 \mathrm{GeV}$ [5]. 


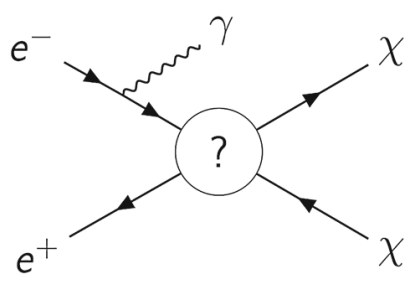

Fig. 1 Diagram describing DM particle pair production process with additional ISR photon radiation

For proper estimate of the experimental sensitivity, precise modelling of all background processes is required.

The WHIZARD program $[6,7]$ is a flexible tool, which is widely used for numerical cross section calculations and generation of Monte Carlo event samples in collider studies for Standard Model processes and many different Beyond Standard Model (BSM) scenarios. The main goal of the presented study is to develop the proper framework for simulation of SM background processes to mono-photon study in WHIZARD. Main SM background contributions are expected to come from the radiative neutrino pair production process

$\mathrm{e}^{+} \mathrm{e}^{-} \rightarrow \nu v \gamma$

shown in Fig. 2, which can not be distinguished from the signal process on the detector level, and the radiative Bhabha scattering

$\mathrm{e}^{+} \mathrm{e}^{-} \rightarrow \mathrm{e}^{+} \mathrm{e}^{-} \gamma$

which contribute to the mono-photon background when both electrons escape undetected along the beam pipe [5].

The standard procedure to take ISR effects into account when generating events with WHIZARD is to use the builtin lepton ISR structure function which includes all orders of soft and soft-collinear photons as well as up to the third order in high-energy collinear photons. However, this approach allows only for a proper modelling of the kinematics of the hard scattering, but is not suitable when we expect photons to be detected in the experiment. The ISR photons generated by WHIZARD should not be considered as ordinary final state particles. Their energy and transverse momenta correspond to the sum over all photons radiated in the event from a given lepton line. For proper description of the photon measurement, the hard non-collinear photon emission should be included in the generation of the considered background process on the matrix element level. Generator-level cuts can be applied, corresponding to the detector acceptance, on the final state photon(s), which should also allow to remove divergences in the cross section calculations. To avoid double-counting, a dedicated merging procedure is then used to remove events with photons from ISR structure function emitted in the same kinematic region. The idea of merging tree-level cross section calculations for processes with different photon multiplicities has been considered in the past, see eg. [8,9].

We present results obtained for the (radiative) neutrino pair production and for the (radiative) Bhabha scattering, for the energy range from $240 \mathrm{GeV}$ to $3 \mathrm{TeV}$, corresponding to the energy reach of the considered future $\mathrm{e}^{+} \mathrm{e}^{-}$collider projects: CEPC [10], FCCee [11], ILC [12] and CLIC [13]. The proposed approach is more general and can be applied to any process with photon radiation, which can be simulated in WHIZARD. Conceptually the procedure is reminiscent of the MLM matching in QCD [14], with simplifications of not including a QED shower, an $\alpha_{e m}$ running, $\gamma^{*} \rightarrow f \bar{f}$ splittings in extra emissions or photons in the initial state, which are justified by the smallness of the coupling, $\alpha_{e m} \ll 1$.

\section{Hard photons from $v \bar{v}$ events}

\section{$2.1 \nu \bar{v}$ production}

Two SM diagrams contribute to the neutrino pair production in $\mathrm{e}^{+} \mathrm{e}^{-}$collisions: the one with s-channel $\mathrm{Z}$ boson production and t-channel exchange of the $\mathrm{W}^{ \pm}$boson. For both processes, the ISR photon can be emitted by the incoming electron or positron, but for the process with the $\mathrm{W}^{ \pm}$boson exchange, radiation is also possible from the $\mathrm{W}^{ \pm}$line, see Fig. 2. This is an additional argument to conclude that the standard approach to ISR photon generation, as implemented in WHIZARD, should not be used for detailed simulation of radiative neutrino pair production events. Contribution of the diagram with photon radiation from the $\mathrm{W}^{ \pm}$line significantly reduces the cross sections for radiative neutrino pair production ${ }^{1}$ with the largest impact observed for multi-photon events and events with high photon transverse momenta. Therefore, for precise kinematic description of photons entering the detector, we need to include hard photon emission directly in the process matrix element (ME) calculation. On the other hand, very soft and collinear photons should still be simulated with the parametric approach, taking into account proper summation of higher order corrections. That is why a dedicated procedure for merging between the two regimes is needed.

\subsection{ME-ISR merging}

In the presented study we use the following variables, calculated separately for each emitted photon, to describe kinematics of the emission:

\footnotetext{
1 This was verified by switching off the $\mathrm{W}^{+} \mathrm{W}^{-} \gamma$ vertex in WHIZARD process definition. Note that the results obtained without this vertex are gauge dependent.
} 
Fig. 2 Diagrams describing the neutrino pair production process with additional photon radiation

Fig. 3 Detector acceptance expected for the future experiments at $\mathrm{e}^{+} \mathrm{e}^{-}$colliders in the $\left(q_{+}, q_{-}\right)$plane, for collision energies of $380 \mathrm{GeV}$ (left) and $3 \mathrm{TeV}$ (right). Dashed lines indicate a default cut used to restrict the phase space for $\mathrm{ME}$ photon generation (red) and the cut used for hard photon selection (black), see text for details
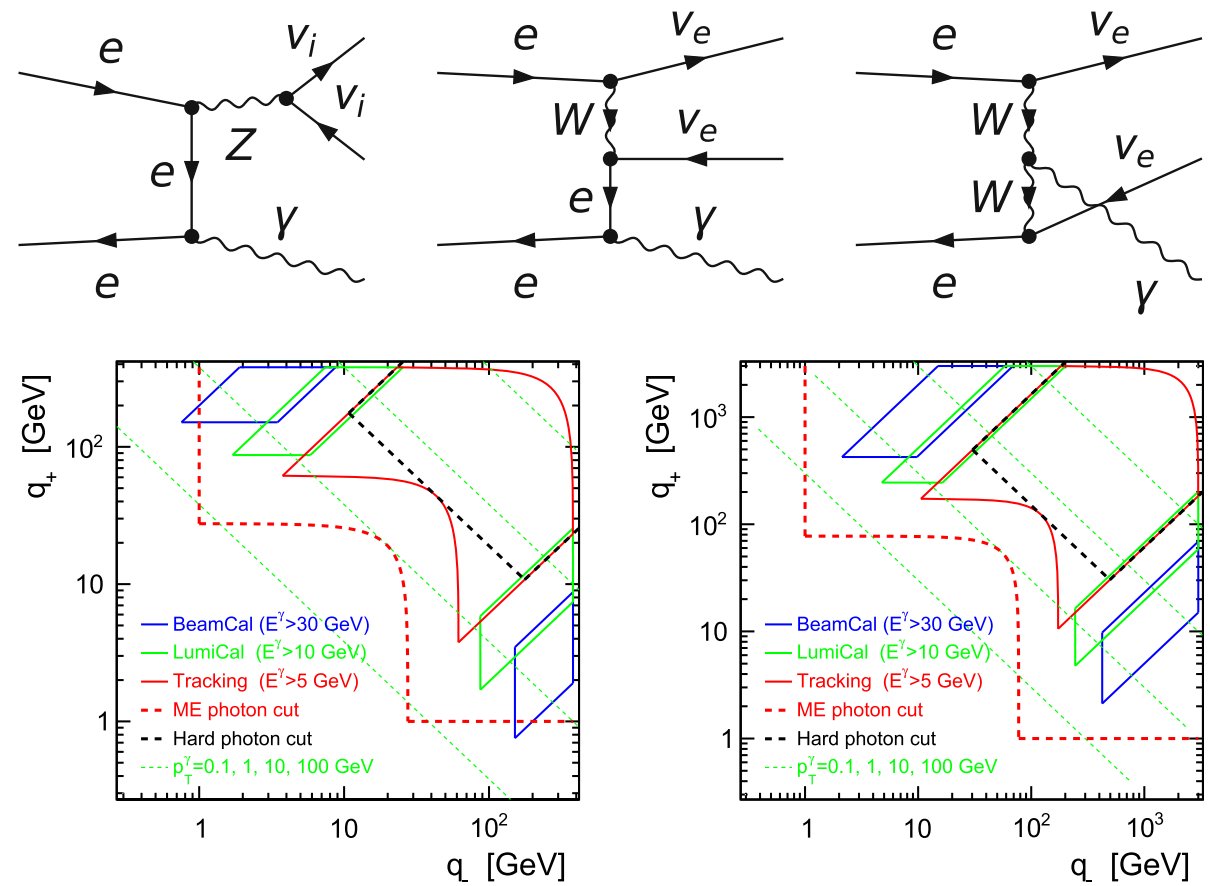

$q_{-}=\sqrt{4 E_{0} E_{\gamma}} \cdot \sin \frac{\theta_{\gamma}}{2}$,

$q_{+}=\sqrt{4 E_{0} E_{\gamma}} \cdot \cos \frac{\theta_{\gamma}}{2}$,

where $E_{0}$ is the nominal $\mathrm{e}^{+}$and $\mathrm{e}^{-}$beam energy, while $E_{\gamma}$ and $\theta_{\gamma}$ are the energy and scattering angle of the emitted photon in question. For the single photon emission they would correspond to the virtuality of the electron or positron after (real) photon emission.

Variables $q_{-}$and $q_{+}$are independent and the pair of values $\left(q_{-}, q_{+}\right)$gives the information on both the energy and scattering angle of a given photon. Shown in Fig. 3 is the expected CLIC detector coverage in the $\left(q_{-}, q_{+}\right)$space, for $380 \mathrm{GeV}$ and $3 \mathrm{TeV}$ running. We assume that photons will be well reconstructed in the detector for scattering angles between $7^{\circ}$ and $173^{\circ}$ (corresponding to the full efficiency of the central tracking detectors, required to reject electron background) and above the energy threshold of $5 \mathrm{GeV}$. Also indicated are the expected acceptances of the beam calorimeter (BeamCal: angular coverage from 10 to $46 \mathrm{mrad}$, energy threshold of $30 \mathrm{GeV}$ ) and luminosity calorimeter (LumiCal: angular coverage 39 to $134 \mathrm{mrad}$, energy threshold of 10 $\mathrm{GeV}$ ) [15]. Only photons with large values of virtualities $q_{-}$and $q_{+}$can be measured in the detector. We therefore require photons generated at the ME level to have energy above $E_{\text {min }}=1 \mathrm{GeV}$ and $q_{ \pm}$virtualities above the merging scale $q_{\min }$. ISR is also taken into account in the cross section integration and generating events, always resulting in two additional photons in the event. ${ }^{2}$ The transverse momenta of ISR photons are taken into account on the event simulation level. ${ }^{3}$ At the same time, to avoid double counting, we reject the events with any of the ISR photons passing the ME photon selection cuts. ${ }^{4}$ This procedure will be referred to as 'ISR rejection' in the following. After ISR rejection, the phase space for photon radiation is unambiguously divided into ME emission and ISR regions, as indicated with red dashed line in Fig. 3 for merging scale $q_{\min }=1 \mathrm{GeV}$. As mentioned above, photons generated by WHIZARD from the ISR structure function should not be considered as single physical particles, but correspond to the sum over all photons radiated in the event from a given lepton line. Therefore, the proposed merging procedure is only approximate.

For the results presented in this paper, version 2.8 .2 of WHIZARD [6,7] was used, compiled with extended precision option. ${ }^{5}$ Shown in Table 1 are the cross sections for neutrino pair production, for different multiplicities of ME photons

\footnotetext{
2 WHIZARD always puts the two ISR photons first in the event record. This allows for easy separation of ME and ISR photons in WHIZARD cuts and event selection, see Appendix A.1 for an example of Sindarin scripts.

3 Implemented ISR handler is used with parameter \$isr_handler_mode = "recoil".

4 The WHIZARD parameter isr_q_max should in principle allow to limit the kinematic range of the ISR emission. However, we noticed that it does not affect the cross section integration so the calculated cross section values can not be used for normalisation of the generated event samples. That is why we have to use the ISR rejection procedure in WHIZARD instead.

5 Extended precision was crucial for the convergence of the Bhabha cross section integration at highest collision energies.
} 
Table 1 Cross section values for (radiative) $\nu \bar{\nu}$ events with different collision energies and different multiplicities of photons included in the matrix element calculations. Number of significant digits indicates the statistical precision resulting from WHIZARD integration

\begin{tabular}{llllll}
\hline$\sqrt{s}[\mathrm{GeV}]$ & \multicolumn{2}{l}{ Whizard-2.8 $\sigma\left(\mathrm{e}^{+} \mathrm{e}^{-} \rightarrow \nu \bar{\nu}\right)[\mathrm{fb}]$} & & $\sigma\left(\mathrm{e}^{+} \mathrm{e}^{-} \rightarrow \nu \bar{\nu}\right)[\mathrm{fb}] \mathrm{after} \mathrm{ISR} \mathrm{rejection}$ \\
\cline { 2 - 5 } & $\nu \bar{\nu}$ & $\nu \bar{\nu}+\gamma_{M E}$ & $\nu \bar{\nu}+2 \gamma_{M E}$ & $\nu \bar{\nu}+3 \gamma_{M E}$ & \\
\hline 240 & 53,900 & 12,600 & 1300 & 64 & 53,800 \\
250 & 53,300 & 12,200 & 1300 & 60 & 53,000 \\
380 & 50,900 & 10,900 & 1200 & 55 & 50,600 \\
500 & 51,200 & 11,300 & 1200 & 75 & 51,200 \\
1000 & 52,800 & 13,600 & 1700 & 120 & 52,200 \\
1500 & 53,300 & 15,400 & 2200 & 170 & 52,100 \\
3000 & 53,900 & 18,500 & 3100 & 180 & 52,400
\end{tabular}

Table 2 Cross section values for different merging parameter $q_{\min }$ in (radiative) $\nu \bar{v}$ events for different multiplicities of photons included in the matrix element calculations. Number of significant digits indicates the statistical precision resulting from WHIZARD integration

\begin{tabular}{|c|c|c|c|c|c|c|}
\hline \multirow[t]{2}{*}{$\sqrt{s}[\mathrm{GeV}]$} & \multirow[t]{2}{*}{$q_{\min }[\mathrm{GeV}]$} & \multicolumn{4}{|c|}{ Whizard-2.8 $\sigma\left(\mathrm{e}^{+} \mathrm{e}^{-} \rightarrow v \bar{\nu}\right)[\mathrm{fb}]$} & \multirow[t]{2}{*}{$\sigma\left(\mathrm{e}^{+} \mathrm{e}^{-} \rightarrow \nu \bar{\nu}\right)[\mathrm{fb}]$ after ISR rejection } \\
\hline & & $\nu \bar{\nu}$ & $\nu \bar{\nu}+\gamma_{M E}$ & $\nu \bar{\nu}+2 \gamma_{M E}$ & $\nu \bar{\nu}+3 \gamma_{M E}$ & \\
\hline \multirow[t]{6}{*}{380} & $q_{\min }=0.1$ & \multirow{6}{*}{50,900} & 16,500 & 2600 & 220 & 50,000 \\
\hline & $q_{\min }=0.5$ & & 12,600 & 1600 & 110 & 50,400 \\
\hline & $q_{\min }=1$ & & 10,900 & 1200 & 55 & 50,600 \\
\hline & $q_{\min }=5$ & & 7000 & 480 & 15 & 50,700 \\
\hline & $q_{\min }=10$ & & 5300 & 270 & 7 & 50,800 \\
\hline & $q_{\min }=50$ & & 1400 & 21 & 0 & 50,500 \\
\hline \multirow[t]{6}{*}{3000} & $q_{\min }=0.1$ & \multirow{6}{*}{53,900} & 26,200 & 6300 & 970 & 52,200 \\
\hline & $q_{\min }=0.5$ & & 20,700 & 4000 & 410 & 52,300 \\
\hline & $q_{\min }=1$ & & 18,500 & 3100 & 180 & 52,400 \\
\hline & $q_{\min }=5$ & & 13,000 & 1600 & 74 & 52,700 \\
\hline & $q_{\min }=10$ & & 10,700 & 1100 & 57 & 52,600 \\
\hline & $q_{\min }=50$ & & 5200 & 260 & 2 & 52,800 \\
\hline
\end{tabular}

(up to three) and different collision energies. The statistical uncertainties resulting from the WHIZARD integration are below or of the order of one permille for processes without or with one ME photon and increase up to 5\% for processes with three photons. The second column gives the cross section for neutrino pair production with ISR photon radiation (before ISR rejection). The effect of the ISR is largest at low collision energies, where it increases the cross section by up to $25 \%$ for $240 \mathrm{GeV}$. The contribution from the 'radiative return' results in the increase of the cross section for the lowest energies. The ISR contribution becomes negligible at the highest collision energies, where the neutrino pair production process is dominated by the $\mathrm{W}^{ \pm}$exchange diagram.

The last column in Table 1 gives the total cross section (sum over photon multiplicities) expected after ISR-ME merging, i.e. after removing events with ISR photons passing the ME photon selection (ISR rejection). The total cross section after ISR-ME merging is very close to the cross section for the $\mathrm{e}^{+} \mathrm{e}^{-} \rightarrow \nu \bar{\nu}$ process with ISR generation only (without ISR rejection) showing that the merging procedure does not affect the normalisation on the sample. Shown in Table 2 are the cross section values for two selected collision energies, $380 \mathrm{GeV}$ and $3 \mathrm{TeV}$, but for different merging scales $q_{\min }$. Cross sections for different photon multiplicities strongly depend on the $q_{\min }$ parameter. However, the total cross section after ISR rejection is basically independent of this parameter. We take $q_{\min }=1 \mathrm{GeV}$ as the default value for the merging parameter in the following.

\subsection{Hard photon selection}

Only for a small fraction of neutrino pair production events the radiated photon can be measured in the detector. Therefore, additional photon selection on the generator level is required for efficient simulation of mono-photon events. We assume the final signal selection will require photon to be reconstructed in the angular range $7^{\circ}<\theta^{\gamma}<173^{\circ}$ and with the transverse momentum $p_{T}^{\gamma}>p_{T}^{\min }=5 \mathrm{GeV}$. These requirements will be referred to as 'hard photon selection' in the following and ME photons passing this selection will 
Table 3 Cross section values for (radiative) $\nu \bar{v}$ events, for different collision energies and different multiplicities of photons included in matrix element calculations with a requirement of at least one of the matrix elements photons to be 'hard'. Number of significant digits indicates the statistical precision resulting from WHIZARD integration

\begin{tabular}{llccc}
\hline$\sqrt{s}[\mathrm{GeV}]$ & \multicolumn{2}{l}{ Whizard-2.8 $\sigma\left(\mathrm{e}^{+} \mathrm{e}^{-} \rightarrow \nu \bar{\nu}\right)[\mathrm{fb}]$} & $\sigma\left(\mathrm{e}^{+} \mathrm{e}^{-} \rightarrow \nu \bar{\nu}\right)[\mathrm{fb}] \mathrm{after}$ ISR rejection \\
\cline { 2 - 5 } & $\nu \bar{\nu}+\gamma_{M E}$ & $\nu \bar{\nu}+2 \gamma_{M E}$ & 37 & 4100 \\
\hline 240 & 4200 & 730 & 48 & 3900 \\
250 & 4100 & 570 & 43 & 3100 \\
380 & 3200 & 590 & 48 & 3000 \\
500 & 3100 & 780 & 74 & 3300 \\
1000 & 3500 & 940 & 98 & 3600 \\
1500 & 3800 & 1300 & 140 & 3900 \\
3000 & 4200 & &
\end{tabular}

Table 4 Cross section values for different merging parameter $q_{\min }$ and different multiplicities of photons included in the matrix element calculations in (radiative) $\nu \bar{\nu}$ events with the 'hard photon' requirement. Number of significant digits indicates the statistical precision resulting from WHIZARD integration

\begin{tabular}{|c|c|c|c|c|c|}
\hline \multirow[t]{2}{*}{$\sqrt{s}[\mathrm{GeV}]$} & \multirow[t]{2}{*}{$q_{\min }[\mathrm{GeV}]$} & \multicolumn{3}{|c|}{ Whizard-2.8 $\sigma\left(\mathrm{e}^{+} \mathrm{e}^{-} \rightarrow \nu \bar{\nu}\right)[\mathrm{fb}]$} & \multirow{2}{*}{$\sigma\left(\mathrm{e}^{+} \mathrm{e}^{-} \rightarrow \nu \bar{\nu}\right)[\mathrm{fb}]$ after ISR rejection } \\
\hline & & $\bar{\nu}+\gamma_{M E}$ & $\nu \bar{\nu}+2 \gamma_{M E}$ & $\overline{\nu \bar{\nu}+3 \gamma_{M E}}$ & \\
\hline \multirow[t]{6}{*}{380} & $q_{\min }=0.1$ & 3200 & 910 & 120 & 3000 \\
\hline & $q_{\min }=0.5$ & 3200 & 670 & 55 & 3000 \\
\hline & $q_{\min }=1$ & 3200 & 570 & 43 & 3100 \\
\hline & $q_{\min }=5$ & 3200 & 340 & 10 & 3100 \\
\hline & $q_{\min }=10$ & 3200 & 230 & 6 & 3100 \\
\hline & $q_{\min }=50$ & 1400 & 22 & 0 & 1400 \\
\hline \multirow[t]{6}{*}{3000} & $q_{\min }=0.1$ & 4200 & 1900 & 260 & 3700 \\
\hline & $q_{\min }=0.5$ & 4200 & 1500 & 220 & 3900 \\
\hline & $q_{\min }=1$ & 4200 & 1300 & 140 & 3900 \\
\hline & $q_{\min }=5$ & 4200 & 850 & 66 & 3900 \\
\hline & $q_{\min }=10$ & 4200 & 680 & 31 & 3900 \\
\hline & $q_{\min }=50$ & 4000 & 240 & 2 & 3700 \\
\hline
\end{tabular}

be described as 'hard'. For multi-photon events, at least one ME photon needs to pass the hard photon selection. An example of the corresponding WHIZARD steering file is given in Appendix A.1.

Shown in Table 3 are the cross sections for neutrino pair production with hard photon emission, for different multiplicities of ME photons and different collision energies. The cross sections listed for different photon multiplicities do not include ISR photon rejection requirement. The total cross section expected after the ISR-ME merging procedure is listed in the last column.

Compared in Table 4 are the cross section values obtained for different merging scales $q_{\min }$, for two selected collision energies, $380 \mathrm{GeV}$ and $3 \mathrm{TeV}$. Cross section for single hard photon production $\left(\nu \bar{v}+\gamma_{M E}\right)$ is expected to be independent of $q_{\min }$, as the hard photon selection is more restrictive than the virtuality cuts applied on ME photons. Deviation from this expectation is only observed for $q_{\min }=50 \mathrm{GeV}$, when the virtuality cut enters the kinematic region of the hard photon selection, see Fig. 3.

For processes with two or three photons emitted on ME level, the cross sections strongly depend on the merging scale, as we require only one of the photons to pass hard photon selection cuts. Nevertheless, the total cross section for neutrino pair production with hard photon radiation, after ISR rejection is applied (last column in Table 4) is independent of $q_{\text {min }}$. This confirms that the proposed ISR-ME merging procedure, despite being only approximate, works very well.

The influence of the beamstrahlung on the neutrino pair production turns out to be small. Taking into account the luminosity spectra expected for CLIC running at $380 \mathrm{GeV}$ and $3 \mathrm{TeV}$ resulted in $1.5 \%$ and $-3.5 \%$ change of the total cross sections (after ISR rejection), respectively. The cross section contribution from the process with four ME photons in the final state is at per mille level and was neglected in this study. 
Fig. 4 Distribution of the hard photon transverse momenta for radiative neutrino pair production at $380 \mathrm{GeV}$ (left) and $3 \mathrm{TeV}$ (right) in WHIZARD, for different values of the merging parameter $q_{\min }$. Distributions are normalised to the number of events expected for integrated luminosity of $1 \mathrm{fb}^{-1}$
Fig. 5 Distributions of the number of photons in the neutrino pair production events generated by WHIZARD and the $\mathcal{K} \mathcal{K}$ MC. Compared are multiplicities of all generated photons (upper row) and for those remaining after hard photon selection (lower row), for collision energy of $380 \mathrm{GeV}$ (left) and $3 \mathrm{TeV}$ (right).

Distributions are normalised to the number of events expected for integrated luminosity of $1 \mathrm{fb}^{-1}$
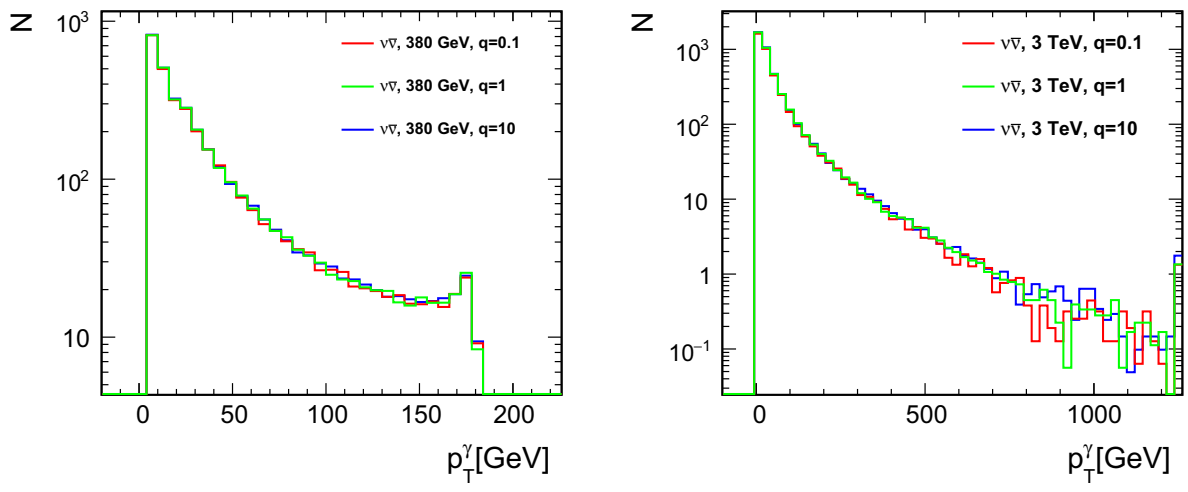

Z

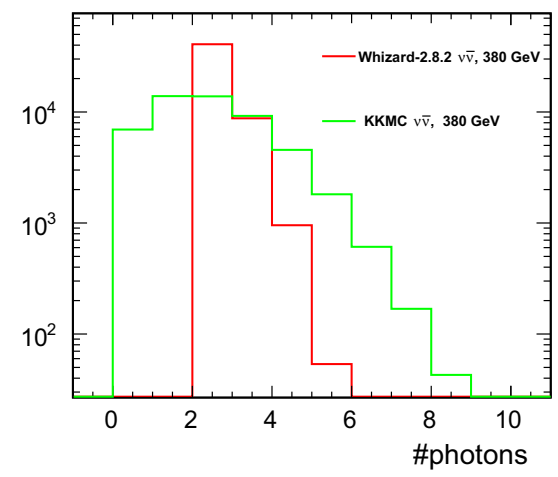

Z

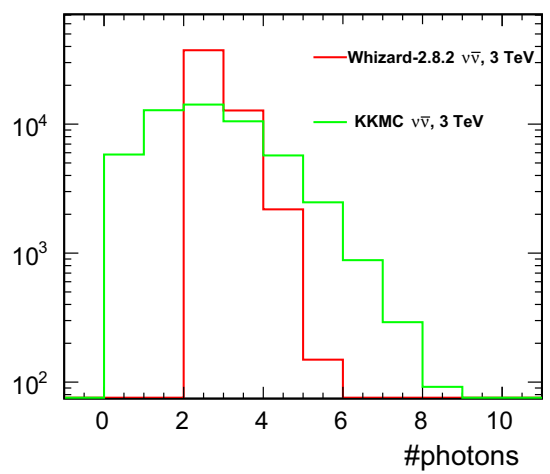

Z

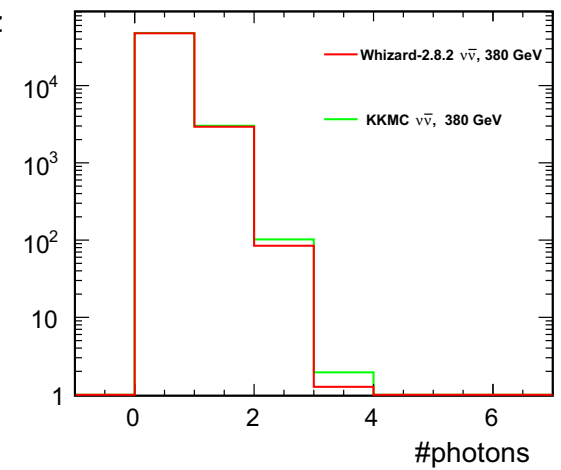

$z$

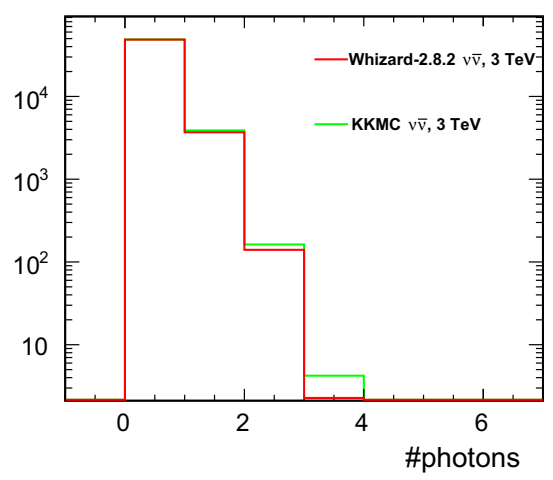

Figure 4 shows the distribution of the hard photon transverse momenta for radiative neutrino pair production at $380 \mathrm{GeV}$ and $3 \mathrm{TeV}$, for different values of the merging parameter $q_{\min }$. It demonstrates that the photon transverse momentum distribution, after hard photon selection, is not sensitive to the choice of the $q_{\min }$ parameter.

\subsection{Comparison of hard photon selection for Whizard and the $\mathcal{K} \mathcal{K}$ MC generators}

To verify the accuracy of the proposed ISR-ME merging procedure we compare the results of WHIZARD 2.8.2 with those of the $\mathcal{K} \mathcal{K}$ MC 4.22 code $[16,17]$. The $\mathcal{K} \mathcal{K}$ MC includes softphoton resummation in Coherent Exclusive Exponentiation (CEEX) [18], based on the Yennie-Frautschi-Suura scheme [19] and the exact $\mathcal{O}\left(\right.$ Born $\left.+\alpha^{3 / 2}\right)$ real and virtual matrix element corrections. Therefore single photon differential distributions in the $\mathcal{K} \mathcal{K}$ MC have a full NLO accuracy. WHIZARD on the other hand includes all order resummation of soft and soft-collinear, and hard-collinear emissions up to 3rd order in the ISR. The proposed procedure supplements WHIZARD's default precision with exact hard matrix elements up to order $\mathcal{O}\left(\right.$ Born $\left.+\alpha^{3 / 2}\right)$. Therefore we expect that observables with at least 2 hard non-collinear photons for processes without FSR should have a similar accuracy as in the $\mathcal{K} \mathcal{K}$ MC while single photon ones will differ by missing genuine 1-loop QED corrections. To allow for direct comparison of the two approaches, electroweak corrections not available in WHIZARD are also disabled in the $\mathcal{K} \mathcal{K}$ MC. These corrections contribute to $2-3 \%$ of the total cross section.

Shown in Fig. 5 are distributions of the generated photon multiplicities, for WHIZARD and the $\mathcal{K} \mathcal{K}$ MC. Respective 
Fig. 6 Distributions of the photon energy (upper row) and transverse momentum (lower row) in the neutrino pair production events generated by WHIZARD and the $\mathcal{K} \mathcal{K} M C$, for collision energy of $380 \mathrm{GeV}$ (left) and $3 \mathrm{TeV}$ (right), after hard photon selection.

Distributions are normalised to the number of events expected for integrated luminosity of $1 \mathrm{fb}^{-1}$ $\mathbf{z}$
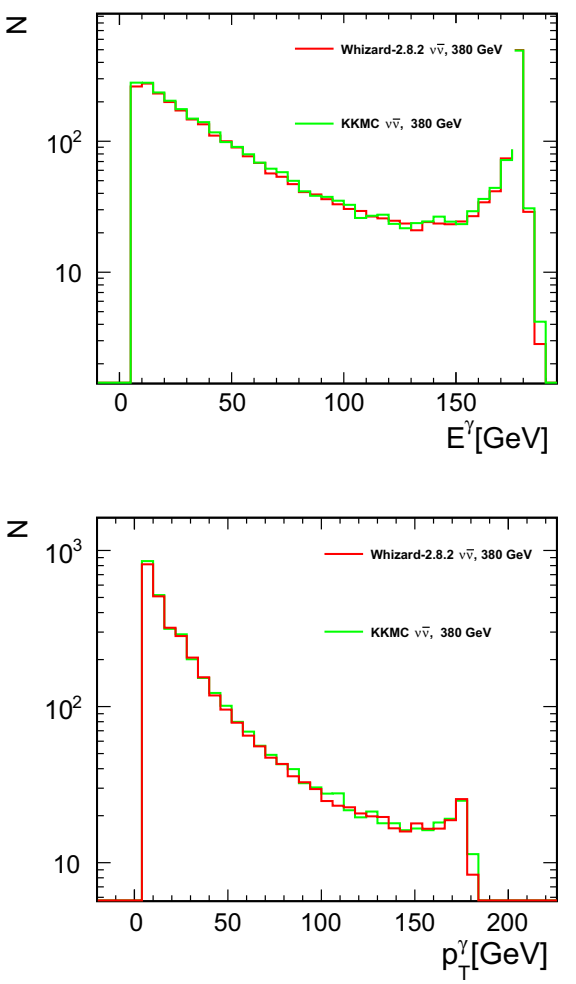

z

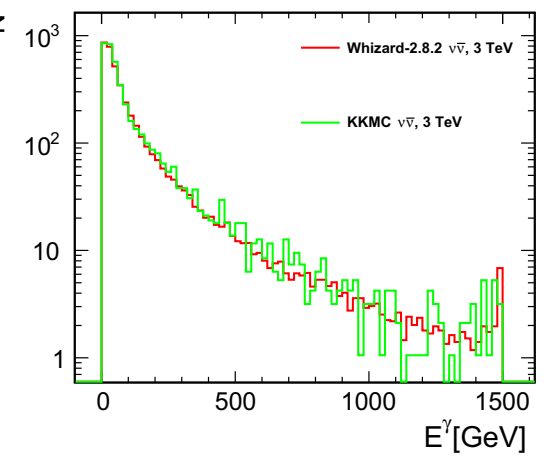

z

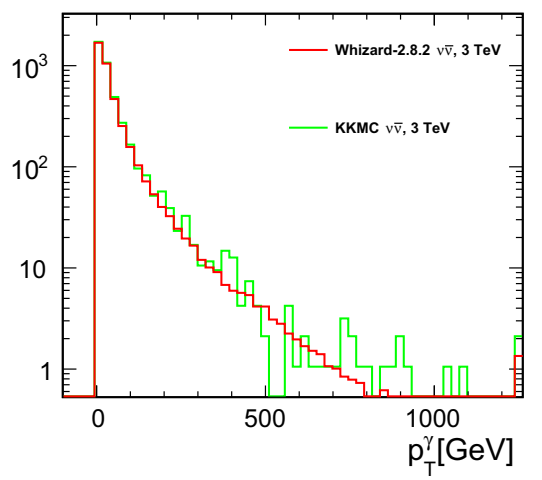

total cross sections for those samples are $50.6 \mathrm{pb}$ and $51.1 \mathrm{pb}$ for the $380 \mathrm{GeV}$ case, $52.4 \mathrm{pb}$ and $52.8 \mathrm{pb}$ for $3 \mathrm{TeV}$ scenario, with errors of order of $0.1 \%$. If all generated photons are considered (upper row in Fig. 5), very different distributions are obtained for the two Monte Carlo codes. The $\mathcal{K} \mathcal{K}$ MC generates events with variable number of photons. Significant fraction of neutrino pair-production events is generated without any photon radiation but there are also events with up to 8 photons in the final state. For WHIZARD, there are always at least two photons in the final state, corresponding to the photons generated from ISR parametrisation for electron and positron beam. Fractions of events with two to five photons correspond to the relative contributions of processes with zero to three photons generated on the ME level. The ME photons generated by WHIZARD need to pass ISR-ME merging criteria and that is why their contribution decreases faster with photon multiplicity than in the case of the $\mathcal{K} \mathcal{K}$ MC.

However, after hard photon selection cuts are applied, corresponding to the expected detector acceptance, the multiplicity distributions obtained with WHIZARD and the $\mathcal{K} \mathcal{K}$ MC are in good agreement. Very good agreement is also observed for photon energy and transverse momentum distributions, which are compared in Fig. 6, for neutrino pair-production at $380 \mathrm{GeV}$ and $3 \mathrm{TeV}$. Total cross sections for $380 \mathrm{GeV}$ samples are $3.1 \mathrm{pb}$ for both generators while for $3 \mathrm{TeV}$ scenario WHIZARD sample has $3.9 \mathrm{pb}$ and $\mathcal{K} \mathcal{K}$ MC $4.1 \mathrm{pb}$. This confirms that the proposed simulation procedure, including ISRME merging gives a proper description of the hard photon production in neutrino pair-production events.

\section{Hard photons from Bhabha events}

When the Bhabha process is to be considered as the background source in the mono-photon analysis, we should not set any constraints on the final state leptons (electron or positron) on the generator level. As they do not need to be observed in the detector, no requirement can be imposed on the minimum momentum transfer or minimum lepton scattering angle. This is the main problem in generation of Bhabha events, as the cross section for this process diverges for low scattering angles due to the Coulomb singularity. However, most of the divergencies are removed when we require a hard photon in the final state, as described in the previous section.

The only requirement which needs to be added, to avoid large collinear enhancement due to the smallness of the electron mass, is the minimum angular separation between final state leptons and photons. Since events with the charged lepton entering the detector together with the hard photon would be anyway rejected in the analysis, this cut should not result in any significant bias in the final analysis results. Angular separation between photons and final state leptons greater than $\theta_{\text {min }}^{e \gamma}=1^{\circ}$ was required for the results presented in this section, see Appendix A.2 for an example of Sindarin steering file for WHIZARD. ${ }^{6}$

\footnotetext{
6 While the choice of the $\theta_{\text {min }}^{e \gamma}$ value does affect the numerical results presented in Tables 5 and 6 (cross section after ISR rejection changes by about $20-30 \%$ when changing the separation cut by an order of magnitude), variations in results obtained after electron rejection cuts are marginal.
} 
Table 5 Cross section values for different collision energies and different multiplicities of photons included in matrix element calculations in Bhabha events with a requirement of at least one of the matrix elements photons to be 'hard'. Number of significant digits indicates the statistical precision resulting from WHIZARD integration

\begin{tabular}{llclc}
\hline$\sqrt{s}[\mathrm{GeV}]$ & \multicolumn{2}{l}{ Whizard-2.8 $\sigma\left(\mathrm{e}^{+} \mathrm{e}^{-} \rightarrow \mathrm{e}^{+} \mathrm{e}^{-}+N \gamma\right)[\mathrm{fb}]$} & \\
\cline { 2 - 4 } & $\mathrm{e}^{+} \mathrm{e}^{-}+\gamma_{M E}$ & $\left.\mathrm{e}^{+} \mathrm{e}^{-}+2 \mathrm{e}^{-} \rightarrow \mathrm{e}^{+} \mathrm{e}^{-}+N \gamma\right)[\mathrm{fb}]$ after ISR rejection \\
\hline 240 & 236,000 & 26,100 & 1500 & 220,000 \\
250 & 224,000 & 24,900 & 1400 & 209,000 \\
380 & 140,000 & 18,400 & 1200 & 128,000 \\
500 & 100,000 & 14,600 & 1100 & 89,800 \\
1000 & 39,800 & 7700 & 700 & 34,400 \\
1500 & 23,000 & 5300 & 500 & 19,400 \\
3000 & 8800 & 2700 & 300 & 7200 \\
\hline
\end{tabular}

Table 6 Cross section values for different merging parameter $q_{\min }$ and different multiplicities of photons included in the matrix element calculations in Bhabha events with a requirement of at least one of the matrix elements photons to be 'hard'. Number of significant digits indicates the statistical precision resulting from WHIZARD integration

\begin{tabular}{|c|c|c|c|c|c|}
\hline \multirow[t]{2}{*}{$\sqrt{s}[\mathrm{GeV}]$} & \multirow[t]{2}{*}{$q_{\min }[\mathrm{GeV}]$} & \multicolumn{3}{|c|}{ Whizard-2.8 $\sigma\left(\mathrm{e}^{+} \mathrm{e}^{-} \rightarrow \mathrm{e}^{+} \mathrm{e}^{-}+N \gamma\right)[\mathrm{fb}]$} & \multirow{2}{*}{$\left.\sigma\left(\mathrm{e}^{+} \mathrm{e}^{-} \rightarrow \mathrm{e}^{+} \mathrm{e}^{-}+N \gamma\right)\right)[\mathrm{fb}]$ after ISR rejection } \\
\hline & & $\mathrm{e}^{+} \mathrm{e}^{-}+\gamma_{M E}$ & $\mathrm{e}^{+} \mathrm{e}^{-}+2 \gamma_{M E}$ & $\mathrm{e}^{+} \mathrm{e}^{-}+3 \gamma_{M E}$ & \\
\hline \multirow[t]{6}{*}{380} & $q_{\min }=0.1$ & 141,000 & 29,500 & 3200 & 123,000 \\
\hline & $q_{\min }=0.5$ & 140,000 & 21,700 & 1500 & 126,000 \\
\hline & $q_{\min }=1$ & 140,000 & 18,400 & 1200 & 128,000 \\
\hline & $q_{\min }=5$ & 141,000 & 10,000 & 400 & 131,000 \\
\hline & $q_{\min }=10$ & 140,000 & 5100 & 170 & 145,000 \\
\hline & $q_{\min }=50$ & 11200 & 160 & 2 & 11000 \\
\hline \multirow[t]{6}{*}{3000} & $q_{\min }=0.1$ & 8900 & 3700 & 420 & 6400 \\
\hline & $q_{\min }=0.5$ & 9000 & 3000 & 230 & 7000 \\
\hline & $q_{\min }=1$ & 8800 & 2700 & 300 & 7200 \\
\hline & $q_{\min }=5$ & 8900 & 2000 & 140 & 7600 \\
\hline & $q_{\min }=10$ & 8900 & 1300 & 90 & 7500 \\
\hline & $q_{\min }=50$ & 7000 & 300 & 10 & 6100 \\
\hline
\end{tabular}

Shown in Table 5 are the cross section values for Bhabha scattering with emission of hard photons, for different collision energies. Compared are cross sections with up to three photons generated on the ME level (at least one of them is required to pass the hard photon selection) and the total cross section, after the ME-ISR merging procedure (ISR rejection cuts), as described in the previous section. Contrary to the neutrino pair-production, Bhabha scattering cross section decreases fast with the increasing collision energy.

Compared in Table 6 are the radiative Bhabha scattering cross sections obtained for different merging scales $q_{\min }$, for two selected collision energies, $380 \mathrm{GeV}$ and $3 \mathrm{TeV}$. As before, the contributions of multiphoton events strongly depend on the merging scale, while the total cross section after ISR rejection is weekly dependent on $q_{\min }$. Only for $q_{\text {min }}=50 \mathrm{GeV}$ (in particular at $380 \mathrm{GeV}$ ) a significant drop of the cross section is observed, as the merging requirement becomes more restrictive than the hard photon selection (see
Fig. 3). Otherwise, cross section values vary by up to $5 \%$ for collision energy of $380 \mathrm{GeV}$ and by up to $11 \%$ for $3 \mathrm{TeV}$, from the values obtained for $q_{\text {min }}=1 \mathrm{GeV}$, when $q_{\text {min }}$ is varied by an order of magnitude. Cross section variation with $q_{\text {min }}$ can be attributed to the contribution from the final state radiation (FSR) and the ISR-FSR interference, which are taken into account in ME approach, but not in the ISR parametrisation. When increasing the $q_{\text {min }}$ cut, the phase space for photons generated with ISR parametrisation is increased while it is reduced for ME generation. This effect should be further reduced when additional selection cuts (in particular electron veto) are applied in the analysis.

The influence of the beamstrahlung on the radiative Bhabha cross section is significant, much larger than for the neutrino pair production. When CLIC luminosity spectra are taken into account, the radiative Bhabha cross section increases by $5 \%$ at $380 \mathrm{GeV}$ and by over a factor of 2 at $3 \mathrm{TeV}$. This is due to the large beamstrahlung effects expected 
Fig. 7 Distribution of the hard photon transverse momenta for radiative Bhabha events at $380 \mathrm{GeV}$ (left) and $3 \mathrm{TeV}$ (right) in WHIZARD, for different values of the merging parameter $q_{\text {min }}$. Distributions are normalised to the number of events expected for integrated luminosity of $1 \mathrm{fb}^{-1}$

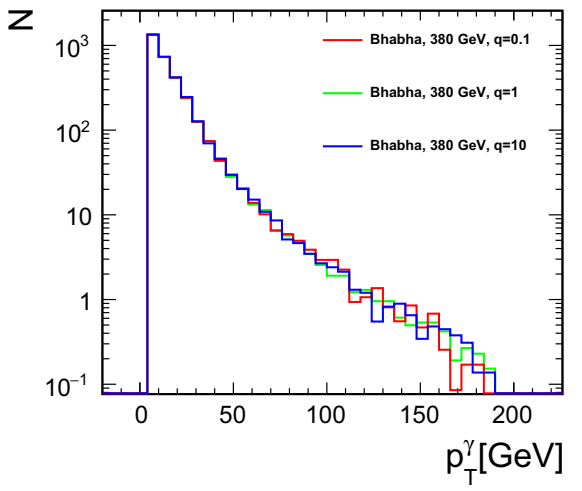

at $3 \mathrm{TeV}$ CLIC and to the Bhabha scattering cross section decreasing rapidly with energy. The cross section contribution from the process with four ME photons in the final state is found to be negligible.

Figure 7 shows the distribution of the hard photon transverse momenta for radiative Bhabha events at $380 \mathrm{GeV}$ and $3 \mathrm{TeV}$, for different values of the merging parameter $q_{\text {min }}$. Also in this case the photon transverse momentum distribution, after hard photon selection, is not sensitive to the choice of the $q_{\text {min }}$ parameter, confirming the validity of the proposed approach.

While the cross section for neutrino pair production with hard photon emission is dominated by events with only a single photon visible in the detector, the situation is very different for the radiative Bhabha scattering. The transverse momentum of the hard photon has to be balanced either by the transverse momentum of the scattered electron or positron, or by emission of additional photons. By applying a veto cut on the energy deposits in the BeamCal and LumiCal detectors, below the acceptance region of the tracking detectors (see Sect. 2.2), about half of the radiative Bhabha scattering events can be identified and rejected. ${ }^{7}$ For events passing such a veto cut, information from the tracking detectors can be used to identify electrons and photons in the central detector region. Expected contributions from different final state topologies are presented in Fig. 8. Samples are clearly dominated by events with scattered electron(s) in the central region. For energies up to $500 \mathrm{GeV}$, Bhabha events with only a single photon visible in the detector $(1 \gamma)$ contribute only to a tiny fraction of the cross section, about $10-20 \mathrm{fb}$. Contribution of events with two photons in the final state is about an order of magnitude higher. Only for the centre-ofmass energy of $1 \mathrm{TeV}$ and above, mono-photon event contribution from Bhabha scattering increases significantly. This is due to the fact, that for the higher beam energy, electrons can balance transverse momentum of the hard photon even when scattered below the BeamCal acceptance. This

\footnotetext{
7 As the tracking information can not be used to distinguish between electrons and photons, the veto cut is also applied to additional ME photons produced in the BeamCal and LumiCal acceptance.
}

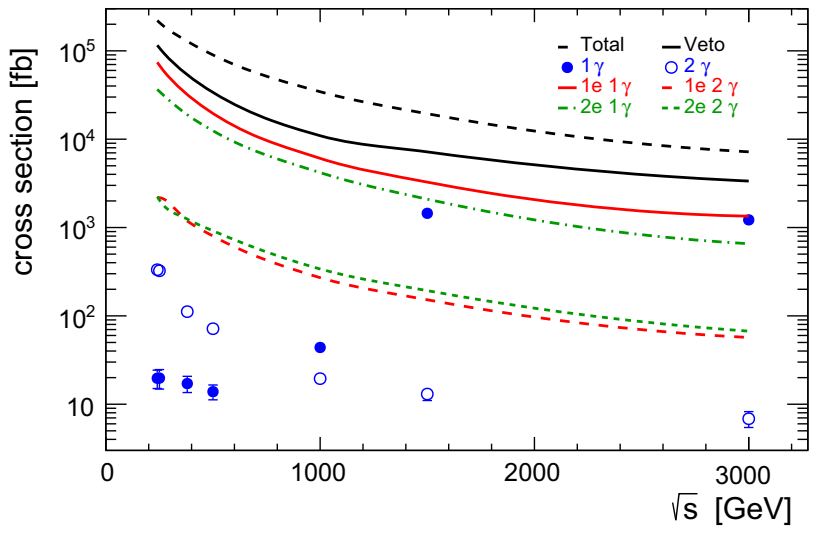

Fig. 8 Cross sections for radiative Bhabha scattering with at least one 'hard' photon, after ISR rejection, as a function of the centre-of-mass energy. Cross sections for different final states observed in the central detector, calculated assuming no activity in the BeamCal or LumiCal detectors, are compared with the overall cross section obtained after applying BeamCal and LumiCal cuts (Veto) and the total cross section for the radiative Bhabha scattering (Total), as given in Table 5

indicates that the minimum photon transverse momentum required in the event selection should probably be increased at the high energy running. Results presented in Fig. 8 indicate also that the detector performance, in particular performance of BeamCal and LumiCal, as well as its modelling in the event simulation, is crucial for the understanding and suppression of the Bhabha background. Also, measurement of events with two reconstructed photons $(2 \gamma)$ can give an important handle for verifying the detector performance and Monte Carlo predictions.

\section{Conclusions}

Production of mono-photon events in $\mathrm{e}^{+} \mathrm{e}^{-}$collisions is considered as a possible signature for many BSM scenarios. Analysis of the energy spectrum and angular distributions of those photons can shed light on the nature of new physics phenomena. Therefore, the precise modelling of the monophoton processes is crucial. In this paper the procedure has been presented which allows for a reliable simulation of the 
mono-photon events in WHIZARD. We focus on the two Standard Model background processes with the same final state: radiative neutrino pair production and radiative Bhabha scattering, and on the energy range considered for the future $\mathrm{e}^{+} \mathrm{e}^{-}$collider projects: from $240 \mathrm{GeV}$ for CEPC up to $3 \mathrm{TeV}$ for CLIC. Presented are cross sections and kinematic distributions of the mono-photon events, based on the proposed merging of the matrix element calculations with the lepton ISR structure function implemented in WHIZARD. Results of such a procedure have been cross-checked with predictions of the $\mathcal{K} \mathcal{K}$ MC generator for neutrino pair production processes. The proposed procedure should be particularly useful for simulation of BSM mono-photon events in WHIZARD. The dominant background contribution is expected to result from the radiative neutrino pair production events, while the background from the Bhabha scattering will very strongly depend on the detector performance, electron veto efficiency in particular.

Acknowledgements We thank J.-J.Blaising, S.Jadach, W. Kilian, J.Reuter and U.Schnoor for valuable comments and suggestions. This contribution was supported by the National Science Centre, Poland, the OPUS project under contract UMO-2017/25/B/ST2/00496 (2018-2021) and the HARMONIA project under contract UMO-2015/18/M/ST2/00518 (2016-2020)

Data Availability Statement This manuscript has no associated data or the data will not be deposited. [Authors' comment: The main purpose of the paper was to present the new approach to mono-photon event simulation with WHIZARD. Presented simulation results were obtained for specific choice of acceptance cuts only to demonstrate the validity of the method. Sindarin files included in the Appendix can be used to reproduce the results or adapt the simulation to the user's needs.]

Open Access This article is licensed under a Creative Commons Attribution 4.0 International License, which permits use, sharing, adaptation, distribution and reproduction in any medium or format, as long as you give appropriate credit to the original author(s) and the source, provide a link to the Creative Commons licence, and indicate if changes were made. The images or other third party material in this article are included in the article's Creative Commons licence, unless indicated otherwise in a credit line to the material. If material is not included in the article's Creative Commons licence and your intended use is not permitted by statutory regulation or exceeds the permitted use, you will need to obtain permission directly from the copyright holder. To view a copy of this licence, visit http://creativecomm ons.org/licenses/by/4.0/.

Funded by SCOAP ${ }^{3}$.

\section{Appendix}

A.1 Sindarin file for generation of radiative $\mathrm{e}^{+} \mathrm{e}^{-} \rightarrow \nu \bar{\nu}$ events
Listing 1 Sindarin file for generation of $\nu \bar{v}(\gamma)$ background

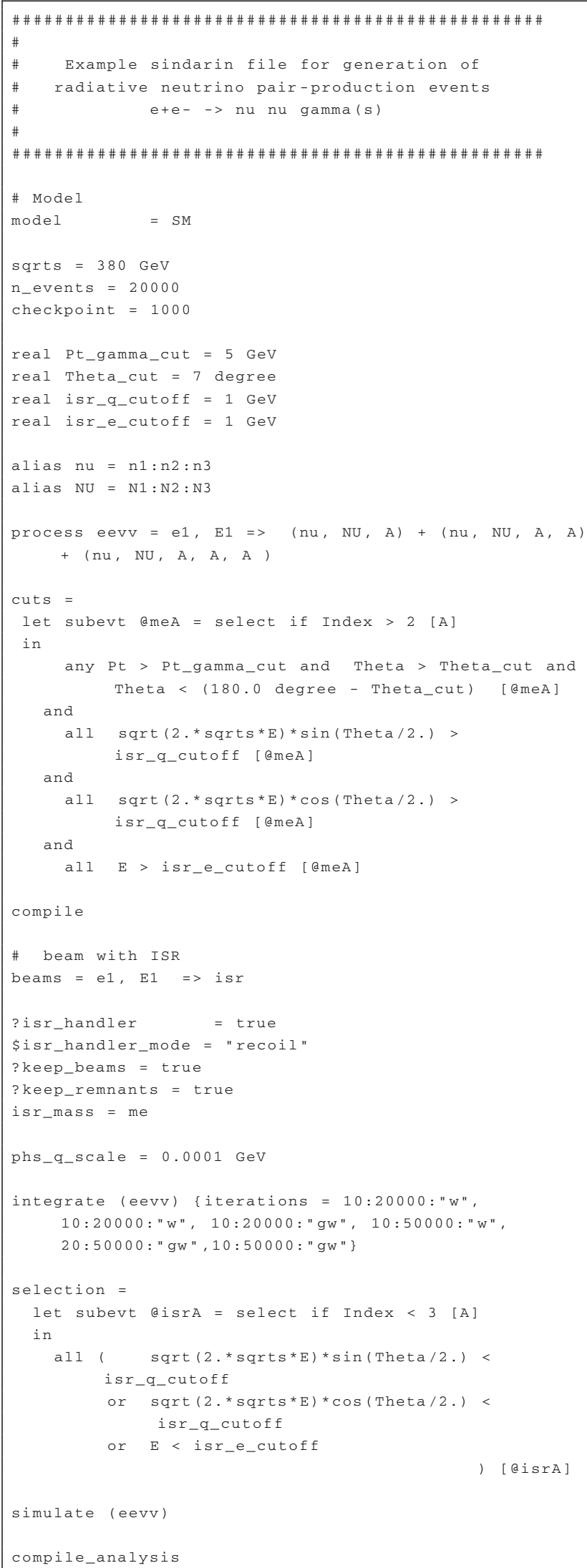

simulate (eevv)

compile_analysis 
A.2 Sindarin file for generation of radiative Bhabha events

Listing 2 Sindarin file for generation of $\mathrm{e}^{+} \mathrm{e}^{-}(\gamma)$ background

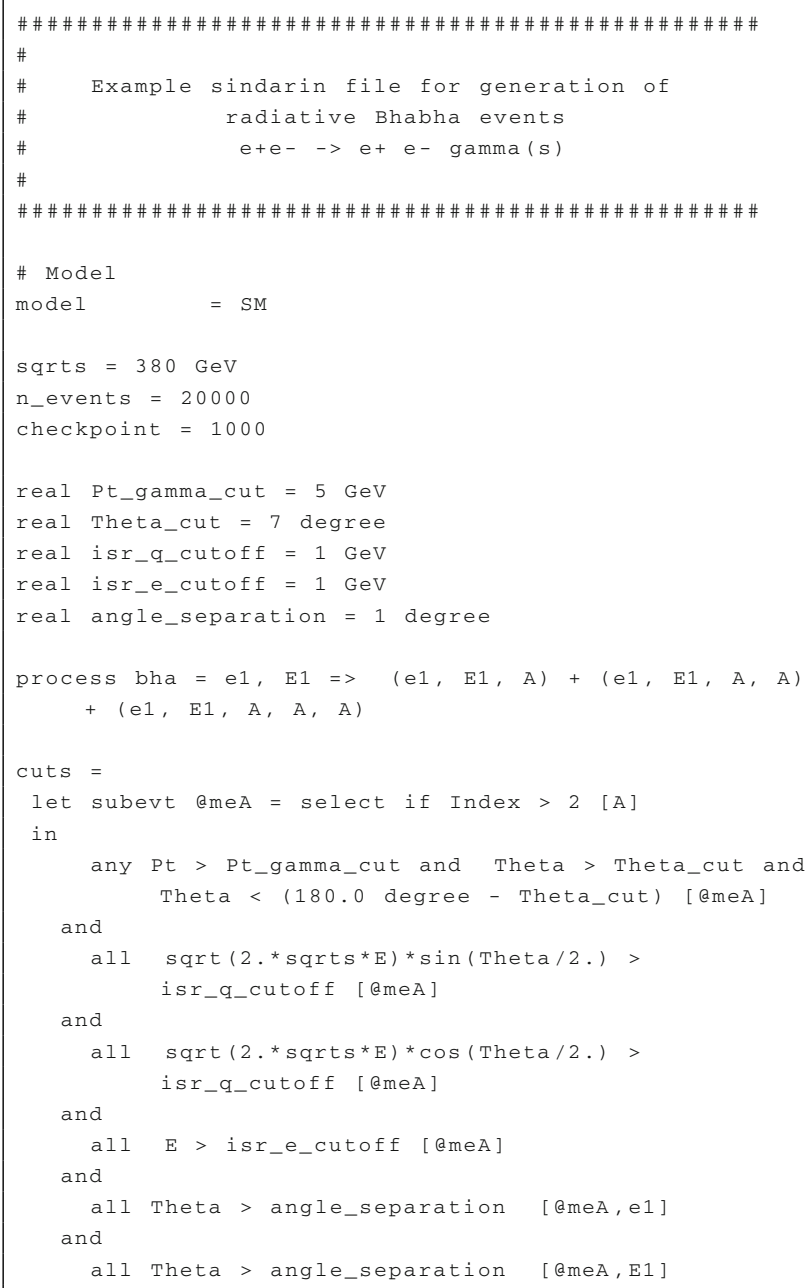

simulate (bha)

compile_analysis

\section{References}

1. P. Achard et al., Single photon and multiphoton events with missing energy in e+e. collisions at LEP. Phys. Lett. B 587, 16 (2004). https://doi.org/10.1016/j.physletb.2004.01.010. arXiv:hep-ex/0402002 [hep-ex]

2. J. Abdallah et al., Photon events with missing energy in e+e. collisions at $\sqrt{s}=130 \mathrm{GeV}$ to $209 \mathrm{GeV}$. Eur. Phys. J. C 38, 395 (2005). https://doi.org/10.1140/epjc/s2004-02051-8. arXiv:hep-ex/0406019 [hep-ex]

3. J. Abdallah et al., Search for one large extra dimension with the DELPHI detector at LEP. Eur. Phys. J. C 60, 17 (2009). https://doi. org/10.1140/epjc/s10052-009-0874-9. arXiv:0901.4486 [hep-ex]

4. P.J. Fox et al., LEP shines light on dark matter. Phys. Rev. D 84, 014028 (2011). https://doi.org/10.1103/PhysRevD.84.014028. arXiv:1103.0240 [hep-ph]

5. M. Habermehl, M. Berggren, J. List, WIMP dark matter at the international linear collider (2020). arXiv:2001.03011 [hep-ex]

6. W. Kilian, T. Ohl, J. Reuter, WHIZARD: Simulating Multi-Particle Processes at LHC and ILC. Eur. Phys. J. C 71, 1742 (2011). https:// doi.org/10.1140/epjc/s10052-011-1742-y. arXiv:0708.4233 [hep$\mathrm{ph}]$

7. M. Moretti, T. Ohl, J. Reuter, O’Mega: An Optimizing matrix element generator (2001) 1981. arXiv:hep-ph/0102195 [hep-ph]

8. G. Montagna et al., Single photon and multiphoton final states with missing energy at $e+e-$ colliders. Nucl. Phys. B 541, 31 (1999). https://doi.org/10.1016/S0550-3213(98)00795-0. arXiv:hep-ph/9807465

9. Y. Kurihara et al., (grc neutrino neutrino gamma) Event generator for the single photon and double photon emission associated with neutrino pair production. Comput. Phys. Commun. 136, 250 (2001). https://doi.org/10.1016/S0010-4655(00)00254-X. arXiv:hep-ph/9908422

10. M. Dong et al., CEPC conceptual design report: volume 2-Physics \& Detector, ed. by J. a. B. Guimarães da Costa et al. (2018). arXiv:1811.10545 [hep-ex]

11. A. Abada et al., FCC Physics Opportunities. Eur. Phys. J. C 79, 474 (2019). https://doi.org/10.1140/epjc/s10052-019-6904-3

12. P. Bambade et al., The international linear collider: a global project (2019). arXiv:1903.01629 [hep-ex]

13. T. K. Charles et al., The compact linear collider (CLIC) 2018 summary report, CERN Yellow Rep. Monogr. 1802, ed. by P. N. Burrows et al. 1 (2018). https://doi.org/10.23731/ CYRM-2018-002, arXiv:1812.06018 [physics.acc-ph]

14. M.L. Mangano et al., Matching matrix elements and shower evolution for top-quark production in hadronic collisions. JHEP 01, 013 (2007). https://doi.org/10.1088/1126-6708/2007/01/013. arXiv:hep-ph/0611129 [hep-ph]

15. D. Arominski et al., A detector for CLIC: main parameters and performance, CLICdp-Note-2018-005 (2018). arXiv:1812.07337 [physics.ins-det]

16. S. Jadach, B.F.L. Ward, Z. Was, The Precision Monte Carlo event generator mathcal $K K$ for two fermion final states in $e^{+} e^{-}$ collisions. Comput. Phys. Commun. 130, 260 (2000). https:// doi.org/10.1016/S0010-4655(00)00048-5. arXiv:hep-ph/9912214 [hep-ph]

17. S. Jadach, B.F.L. Ward, Z. Was, KK MC 422: Coherent exclusive exponentiation of electroweak corrections for $\bar{f} f f^{\prime} f^{-\prime}$ at the LHC 
and muon colliders. Phys. Rev. D 88, 114022 (2013). https://doi. org/10.1103/PhysRevD.88.114022. arXiv:1307.4037 [hep-ph]

18. S. Jadach, B.F.L. Ward, Z. Was, Coherent exclusive exponentiation for precision Monte Carlo calculations. Phys. Rev. D 63, 113009 (2001). https://doi.org/10.1103/PhysRevD.63.113009. arXiv:hep-ph/0006359 [hep-ph]
19. D.R. Yennie, S.C. Frautschi, H. Suura, The infrared divergence phenomena and high-energy processes. Ann. Phys. 13, 379 (1961). https://doi.org/10.1016/0003-4916(61)90151-8 